\title{
Un an dans la vie des Services de santé des Forces canadiennes
}

$\mathrm{N}$ ovembre, c'est le mois du souvenir. Cette année, ce ne sont pas les souvenirs qui manquent pour le personnel militaire des Services de santé, ni pour les Forces canadiennes. Au cours de l'année écoulée, nous avons déménagé notre petit hôpital de campagne de Kaboul, dans le nord de l'Afghanistan, à Kandahar, dans le sud, pour nous joindre à un hôpital américain déjà installé et le remplacer ensuite tout en l'agrandissant pour en faire une installation multinationale tous services avec des partenaires du Royaume-Uni, des Pays-Bas, des États-Unis et du Danemark. Nous avons mis en service pour la première fois un tomodensitomètre et un ensemble complet d'appareils d'imagerie numérique et lancé un service de téléradiologie, tout en recevant et traitant des blessés. Garantir des soins de première classe aux militaires de la coalition depuis l'endroit où ils ont été blessés jusqu'à leur retour chez eux au Canada, c'est une "mission qui ne peut échouer» et notre personnel de santé a relevé le défi. Nous réapprenons de vieilles leçons tout en étant aux prises avec de nouveaux problèmes et en portant le soin des blessés au combat à de nouveaux niveaux d'excellence. Grâce aux améliorations du secourisme de campagne, à l'évacuation par hélicoptère et aux appareils de protection personnelle, plus de blessés arrivent vivants à l'hôpital de campagne. Leurs problèmes sont plus complexes et lorsqu'on a réussi à stabiliser leur état, il faut les évacuer rapidement en passant par l'hôpital de l'Armée de terre américaine en Allemagne. La gestion de l'évacuation aéromédicale de patients aux soins intensifs pour les transporter de l'Allemagne au Canada est un des services que nous assurons actuellement de façon routinière et nous sommes devenus très efficaces à cet égard. Nous y sommes parvenus en dépit de graves pénuries d'effectifs en uniforme et face à la décroissance et à la "privatisation" des services médicaux militaires.

Depuis la fermeture de nos hôpitaux militaires survenue au cours de la dernière décennie, nous redécouvrons aussi nos liens essentiels avec le secteur des soins de santé civils du Canada. Nous avons des cliniciens militaires intégrés à des établissements civils un peu partout au Canada, et nos cliniques militaires emploient beaucoup de prestateurs de soins civils. Les échanges et la compréhension mutuelle qui en découlent nous ont bien servis pendant notre évolution vers un tout nouveau paradigme sur le plan du soin de nos militaires. La coopération et les efforts de nos collègues civils partout au Canada lorsque ces soldats reviennent au pays et qu'ils ont besoin de soins très spéciaux nous font vraiment chaud au cœur.

Ces soldats, ce sont des patients très spéciaux. Je l'ai toujours su, mais le fait d'en voir tellement d'entre eux revenir au pays blessés a poussé ma compréhension à un niveau dif- férent. Ce sont nos enfants, nos conjoints, nos amis; ils et elles s'acquittent de leur devoir envers le Canada de façon exemplaire en acceptant pleinement la responsabilité illimitée d'être exposés au danger, et nous leur devons les meilleurs soins. Jusqu'à maintenant, je crois que nous les avons fournis et je n'ai jamais été plus fière d'être membre des Services de santé des Forces canadiennes.

Le personnel de l'hôpital et les équipes chirurgicales ont accompli régulièrement de petits miracles dans les circonstances les plus difficiles, sauvé beaucoup de vies et de membres et ranimé l'espoir, innové en médecine de campagne et gagné le respect et l'admiration de l'OTAN, de nos partenaires de la coalition et du peuple afghan. Dans mon cas particulier, j'ai trouvé extrêmement enrichissant d'avoir joué un rôle en permettant à nos infirmiers de se déployer avec plus de confiance après avoir reçu une formation à la fine pointe de la technologie et de voir des progrès comme QuikClot, le pansement de combat compressif avancé israélien et le cours de soins des traumatisés au combat, qui sauvent des vies tous les jours. Comme professionnels de la santé, nous faisons tous don de nous-même pour nos patients. Ces infirmiers le font dans les circonstances les plus ardues, dans les mêmes conditions et exposés au même danger que nos fantassins, et leurs camarades de combat les vénèrent. Ce sont eux qui permettent à l'hôpital de campagne d'accomplir des miracles parce qu'ils y amènent les blessés vivants. Nous remercions du plus profond du cœur le cpl Eykelenboom et le cpl Arnold, nos premiers infirmiers tombés au combat depuis la Guerre de Corée, ainsi que le cpl Franklin, qui a perdu les deux jambes dans une attaque suicide et qui est maintenant affairé à transmettre son savoir-faire à d'autres infirmiers : vous avez fait le sacrifice ultime au service de vos patients et de votre pays et incarnent les plus grands idéaux du service. Vous êtes une source d'inspiration pour nous tous.

En terminant, je veux remercier, au nom du commodore Kavanagh, notre directeur général des Services de santé, et du brigadier-général Jaeger, notre chirurgien général, tous les fournisseurs civils de soins de santé qui nous ont tellement aidés en ces temps difficiles. Votre appui a joué un rôle central et continuera de le faire. Le Canada a un des meilleurs systèmes de santé au monde; il a démontré qu'il est prêt à nous aider en période de besoin et qu'il peut le faire.

\section{Maureen Haberstock}

Opérations des Services de santé

Forces canadiennes

Ottawa (Ont.)

Cet article a fait l'objet d'un examen par les pairs. 